\title{
QUALITY OF SERVICE MODEL AND SIGNALING FOR CELLULAR IP ACCESS NETWORK
}

\author{
Suraj Jaiswal, ${ }^{1}$ Fernando Boavida, ${ }^{2}$ Joao Orvalho ${ }^{2,3}$ and Sukumar Nandi ${ }^{1}$ \\ ${ }^{1}$ Department of Computer Science \& Engineering \\ Indian Institute of Technology \\ Guwahati, India \\ \{suraj, sukumar\}@iitg.ernet.in \\ ${ }^{2}$ Department of Informatics Engineering \\ University of Coimbra, Portugal \\ boavida@dei.uc.pt \\ ${ }^{3}$ Polytechnic Institute of Coimbra, Portugal \\ orvalho@esec.pt
}

\begin{abstract}
There has been a recent trend towards an all-IP network as it provides for costsavings together with optimized network and ease of use. IP based networks are fully utilized, applications can share the same connection and QoS levels can still be guaranteed. But IP was designed for wired networks and wireless scenario was not envisioned. Owing to explosive growth of wireless users and growing demand of wireless services the internet body IETF has developed mobility management schemes to enhance IP in mobility scenarios for wireless subscribers. This trend has resulted in the development of Mobile IP to support node mobility. Mobile IP is only suitable for macro-mobility hence alternate protocols have been proposed in the literature to support seamless handoff in micro-mobile environments like cellular access networks where mobile nodes change their point of attachment frequently. Cellular IP is one of the popular solutions that support fast handoff with paging in a cellular access network. In this paper, we propose a Quality of Service model architecture based on IntServ for Cellular IP access network and describe the signaling mechanism RSVP integrated with the native protocol used to achieve the desired QoS.
\end{abstract}

Keywords: Access Network, Cellular IP, QoS, IntServ, RSVP.

\section{Introduction}

Recently there has been an exponential growth in mobile communication with the development of affordable mobile computing devices like palmtops and laptops. Mobile users are now able to access the Internet through these 
hand-held devices and want similar services as those of users in the wired network for multimedia and real-time applications like video-conferencing, IP telephony etc. A big drop in service quality as these mobile users move from one region to another may not be acceptable to these applications. The Internet Engineering Task Force has introduced two architecture for quality of service provisioning - the Integrated Services (IntServ [3]) model and Differentiated Services (DiffServ) model. In the IntServ model, network resources are explicitly identified and reserved. Network nodes classify incoming packets and use the reservations to provide QoS. In the DiffServ model, resources are not explicitly reserved. Instead, traffic is differentiated into a set of classes, and network nodes provide priority-based treatment based on these classes.

The IETF IntServ and DiffServ model have been designed to work in wired networks. For these models to work in a wireless/mobile environment they must take into account the chief characteristics of wireless networks viz. low bandwidth, battery power constraints, high bit-error and mobility.

In this paper, we propose a QoS model using IntServ architecture for a wireless access network using Cellular IP ([1],[2]) as a solution for node mobility in the access network at the network layer. Our QoS model uses an enhanced RSVP signaling protocol for efficient resource usage.

\section{Background and Related Work}

\section{Integrated Services}

IntServ (Integrated Services) is a per-flow based QoS framework with dynamic resource reservation. Its fundamental philosophy is that routers need to reserve resources in order to provide quantifiable QoS for specific traffic flows. RSVP (Resource Reservation Protocol) [4] serves as a signaling protocol for applications to reserve network resources.

RSVP adopts a receiver-initiated reservation style which is designed for a multicast environment and accommodates heterogeneous receiver service needs. RSVP works as follows (see Fig. 1). The flow source sends a PATH message to the intended flow receiver(s), specifying the characteristic of the traffic. As the PATH message propagates towards the receiver(s), each network router along the way records path characteristics such as available bandwidth. Upon receiving a PATH message, the receiver responds with a RESV message to request resources along the path recorded in the PATH message in reverse order from the sender to the receiver. Intermediate routers can accept or reject the request of the RESV message. If the request is accepted, link bandwidth and buffer space are allocated for the flow, and the flow-specific state information is installed in the routers. Reservations can be shared along branches of the multicast delivery trees. RSVP takes the soft state approach, which regards the flow-specific reservation state at routers as cached information that is installed 


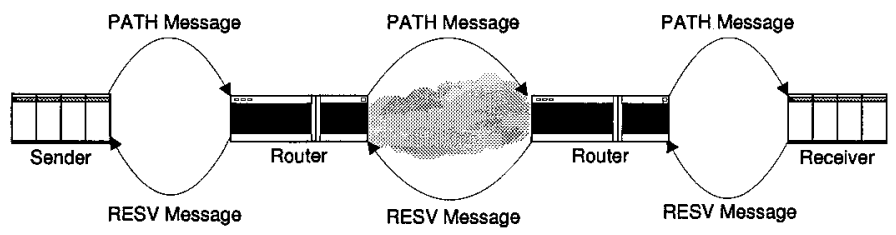

Figure 1. $\quad$ RSVP Signaling.

temporarily and should be periodically refreshed by the end nodes. State that is not refreshed is removed after a timeout period. If the route changes, the refresh messages automatically install the necessary state along the new route. The soft state approach helps RSVP to minimize the complexity of connection setup and improves robustness, but it can lead to increased flow setup times and message overhead.

The IntServ architecture adds two service classes to the existing best-effort model, guaranteed service and controlled load service. Guaranteed service provides an upper bound on end-to-end queuing delay. This service model is aimed to support applications with hard real-time requirements. Controlledload service provides a quality of service similar to best-effort service in an underutilized network, with almost no loss and delay. It is aimed to share the aggregate bandwidth among multiple traffic streams in a controlled way under overload condition. By using per-flow resource reservation, IntServ can deliver fine-grained QoS guarantees. However, introducing flow-specific state in the routers represents a fundamental change to the current Internet architecture. Particularly in the Internet backbone, where a hundred thousand flows may be present, this may be difficult to manage, as a router may need to maintain a separate queue for each flow. Although RSVP can be extended to reserve resources for aggregation of flows, many people in the Internet community believe that IntServ framework is more suitable for intra-domain QoS or for specialized applications such as high-bandwidth flows.

\section{Cellular IP}

Cellular IP (CIP) is a micro-mobility protocol developed for wireless access network. A Cellular IP access network connects to the internet via a domain gateway router. A mobile node attached to the access network will use the IP address of the gateway as its Mobile IP care-of-address. The main component of the CIP networks is the base station. The base stations are used as wireless access points by the mobile node to send and receive data.

Cellular IP gateway periodically broadcasts a beacon packet that is flooded in the access network. Base stations record the neighbor they last received this beacon from and use it to route packet towards the gateway. All packets trans- 
mitted by the mobile node regardless of their destination are routed towards the gateway using these routes. The entry in the routing table is of the form (destination_addr, interface_num) where interface number is the interface on which to forward the packet. Note that CIP routing is based on the interface links rather than on IP address of the next hop as in normal IP routing.

After a mobile node powers up, it informs the gateway router about its current point of attachment by means of a route-update control packet. This packet received by the base station is forwarded towards the gateway on a hop-by-hop basis along the shortest path. The network interface link used by a node to forward the packet towards the gateway is called the uplink of the node and all other links attached to the node are called downlinks. Each CIP node maintains a routing cache in which it stores the mobile node specific routes. The path taken by the packets from mobile node towards the gateway are cached by all the intermediate nodes. To route packets destined for the mobile node the nodes use the reverse of path used by recently transmitted packets from the mobile node which are present in the routing cache. The CIP nodes forward packets either along the uplink interface or along one of the downlink interface.

CIP allows the mobile nodes to be in two states. When they are actively transmitting or receiving packets they are in active state. In active state the mobiles periodically send update packets towards the gateway to keep up-todate location information about itself in the routing cache. Otherwise, they are in idle state or power saving mode. Due to lack of updates, the soft-state routing cache mappings of idle mobile hosts times out. In order to remain reachable in this mode, the mobile sends paging-update packet about its location at regular intervals defined by paging-update-time and these updates are kept in a separate cache called paging cache in certain nodes.

In CIP, a mobile node initiates a handoff based on signal strength measurements. We describe the semi soft handoff mechanisms of CIP which minimizes packet loss.

Semi-soft Handoff. Semi-soft handoff is optimised for CDMA kind of networks. CIP-enabled mobile nodes keep information about the received signal strength from the periodic base station CIP beacon broadcasts. When a mobile node decides to handoff to a new base station based on signal strength measurements, it informs the new base station about the impending handoff while listening to the old base station. The new base station initiates the establishment of a mobile-specific route from the CIP gateway to this new base station so that data packets destined for the mobile host can be sent to the new base station ahead of the actual handoff, while the mobile node keeps on getting data from the old base station during this time. This is called semi-soft handoff. Semi-soft handoff is achieved by sending a semi-soft packet towards the gateway from the mobile node to the new base station. When the semi-soft 


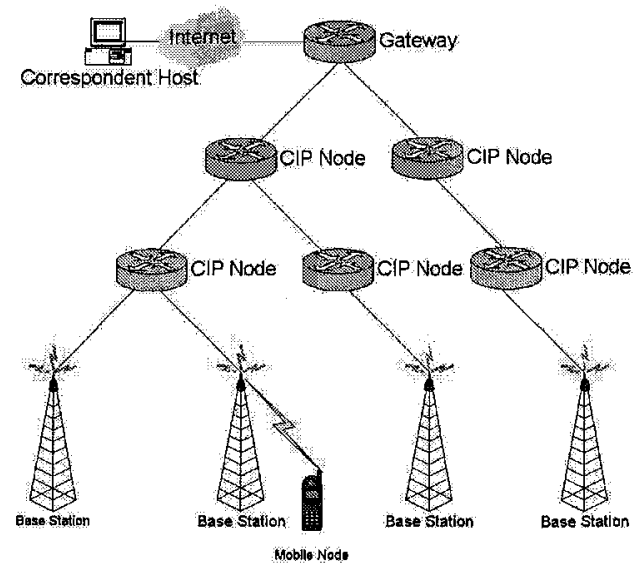

Figure 2. Cellular IP Access Network.

handoff packet reaches a cross over node (so called because it is the common node on the shortest path from the old and new base station to the gateway) the cross over node starts sending the data also towards the new base station. As the semi-soft handoff packet is forwarded towards the gateway, a reverse mapping for the mobile node is created in the CIP-enabled nodes to route data packets towards the mobile node. Thus, the semi-soft packet needs to travel only to the cross over node, which can then start sending data packets to both the old and the new base stations. In the worst case this cross over node can be the gateway.This is all done before the handoff has actually taken place so when the mobile node handoffs it keeps on getting data packets without having to initiate any handover messages, hence preventing any packet loss. Otherwise it would have lost those packets which are forwarded along the old path by the cross over node during the time it takes for the handoff message to reach the cross over node. After handoff is complete the mobile host sends a route update packet towards the gateway which results in the cross over node removing the old entry in the routing table. Fig. 3. illustrates the Semi-soft handoff mechanism.

Introducing Base Station Specific Routes. The base station specific routing table is similar to mobile node specific routing table in the sense that the base station specific routing table contains per base station routing entries. The base stations send routing updates uplink towards the gateway on a hop-by-hop basis just like mobile nodes send route update packets. The routing updates from the base stations when pass through the cellular IP nodes the nodes use it to update the routing entry for that particular base station. A CIP node contains the routing entry for only those base stations whose shortest path to the gate- 


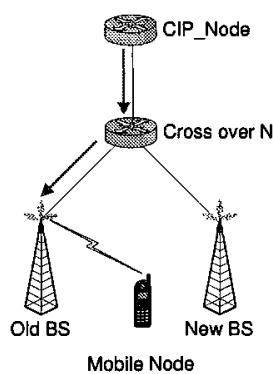

(a) Before Handoft

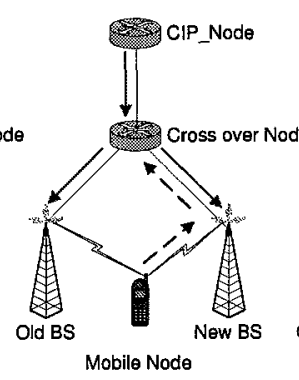

(b) DuringHandoff

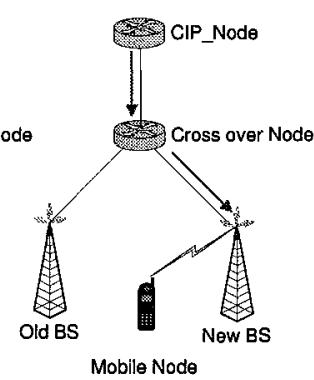

(c) After Handoft

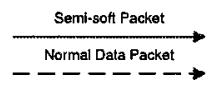

Figure 3. Semi-soft Handoff.

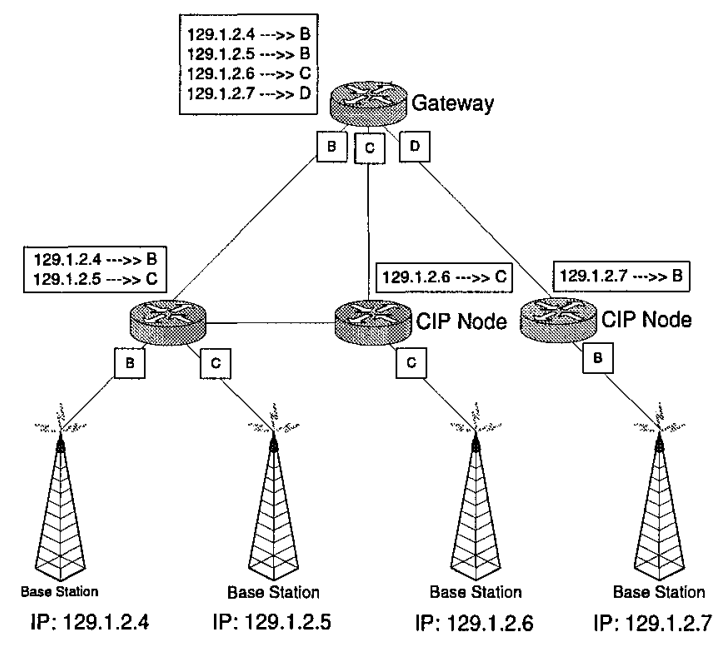

Figure 4. Base Station specifi c routing entries in the Cellular IP nodes.

way contains this cellular IP node, hence this routing table for base stations will be very small (a large wireless access network will have somewhere around a hundred base stations). The timer associated with these routing entries make the routing fault tolerant against base station failures. The base stations send route updates periodically. This period can be in the order of minutes so as not to overload the access network without compromising on the freshness of the routing entries. Fig. 4. makes the idea more clear. The boxes in Fig. 4. shows the entries in the base station specific routing table and alphabet letters denotes the interfaces of a CIP node. 


\section{Related Work}

Recently there has been some work on IntServ in mobile and wireless networks using RSVP. In [5], the architecture for QoS using RSVP in Integrated Services Packet Network has been described, and a resource reservation protocol MRSVP, for mobile nodes has been propose by Talukdar. The main feature of this protocol is the concept of active and passive reservations that is used to provide mobility independent service guarantees. Four additional messages are used in addition to messages already present in RSVP. The drawback of this architecture is that a mobile must know the addresses of all the subnets it is going to move into and which is not always possible. It also places the burden of finding the proxy agents in all these subnets on the mobile. In $[6,7]$, the proposal by Mahadevan also suggests two kinds of resource reservations that contain passive reservation and active reservation. This architecture is based on the assumption that a base station knows the addresses of all the base stations in the neighboring cells, and thus solves the burden placed on the mobile node in [5].

To support the per-flow QoS requirement in a micro mobility environment, [8] proposed the extended RSVP supporting mobility. This scheme makes resource reservation in advance at the locations where it may visit during the lifetime of the connections. But, since [8] does not assume a specific mobility management scheme, the extended RSVP handles not only resource reservation but also mobility management. Therefore the RSVP proposed in [8] requires excessive overhead for the implementation and adaptation of the protocol.

\section{QoS Model and Signaling for Cellular IP Access Network}

In our proposal, we assume IntServ in the Cellular IP access network to guarantee quality of service since it is suitable for access networks where flows of the same class need different and fine grained QoS. Also, we assume that a base station in the Cellular IP access network knows the addresses of the base stations in the neighboring cells. Our work focuses on resource reservation using RSVP in the access network only. Outside the access network, IntServ or DiffServ may be used and the domain gateway provides the necessary mapping as the case may be.

We present the modified RSVP mechanism to reserve network resources in the Cellular IP access network. The changes that need to be made to RSVP include:

- Incorporating passive reservation scheme as in MRSVP [5]. 
- Incorporating QoS parameters specific to mobile environment. These parameters could include loss profiles [9], probability of seamless connection [9], power profile of the mobile node etc.

- Incorporating changes into RSVP PATH state to take into account that Cellular IP uses uplink-downlink (interface on which to send the packet)routing rather than regular next hop (IP address of the next hop) forwarding as in IP routing.

- The RSVP signaling is integrated with Cellular IP signaling to prevent signaling overheads in the access network.

The RSVP module running in the mobile node does not require any change to the existing RSVP module other than to the SPEC object (traffic specification). The mobile node uses MSPEC (mobile specification) object to take into account the characteristics of the wireless link and the limited capability of the mobile node in terms of power etc.

Case I. Mobile Node is in Active State.

Mobile Node is Receiver.

- As the PATH message flows towards the mobile the RSVP daemon in the intermediate Cellular IP nodes create PATH state. This path state contains the PHOP object which describes the previous hop from which this PATH message has come. Here the PHOP always contains the address of the domain gateway. Now, we explain the use of gateway's address in PHOP object. We know that Cellular IP routing table has an entry for the domain gateway. The packet forwarded towards the gateway always takes the uplink route. Hence, we take advantage of this fact and use the gateway's address in PHOP object. Thus PATH state at each node would have previous hop as the gateway and when RSVP messages are forwarded upstream they would always take the uplink route.

- When a mobile node receives PATH message it responds with a RESV message. The RESV message contains the MSPEC object which describes the QoS desired by the mobile node.

- When the base station with which the mobile node is currently attached receives the RESV message from the mobile node:

1 It creates an AGGR_PASV_RESV (aggregate passive reservation) message which needs to be sent towards the base stations in the neighboring cell. For this, it creates a RSVP object class called NEIGHOURS in this message to carry the address of the base station in neighboring cells in 
the aggregate passive reservation message. This is a new object incorporated in RSVP used in the access network.

2 This base station constructs the AGGR_PASV_RESV message which contains all the RSVP class objects necessary to establish the RESV and PATH state in the intermediate nodes. This base station gets the desired objects from its PATH state and the RESV message it receives from the mobile node.

3 The base station sends the RESV and AGGR_PASV_RESV message upstream. The RESV message establishes active reservation from gateway to the base station and the AGGR PASV_RESV message establishes the passive reservation towards the neighboring base stations the mobile node can possibly visit in the future as shown in Fig. 5.

The processing of RESV message, as it is forwarded towards the gateway, is trivial. When the RSVP daemon in a node receives AGGR_PASV_RESV message it gets the list of IP addresses from the NEIGHOURS object. The daemon then asks the routing table if it has entries for these addresses. The daemon communicates with the routing table through an interface such as RSRR (Routing Interface for RSVP) [10]. For the addresses for which the routing table has entry, the RSVP daemon constructs separate UNI_PASV RESV (unicast passive reservation) message to be sent towards these addresses (remember these are base stations and we have base station specific entries in the routing table through base station specific routing implementation) and it removes these entries from the NEIGHOURS object of the AGGR PASV_RESV message. After this, the AGGR_PASV_RESV message is sent upstream. When the RSVP daemon notices an empty NEIGHOURS object in an aggregate passive reservation message it no longer forwards it upstream.

Let's understand this from Fig. 6. In the figure, mobile node is currently in the cell of the second base station. It sends a RESV message towards the base station on receiving a PATH message. This base station then sends an aggregate passive reservation message (its NEIGHOURS object contains the address of the first and third base station) upstream denoted by the red line in the figure. Note that the flow of aggregate passive reservation message does not create any reservation as such. When the CIP node 3 gets this message, its RSVP daemon comes to know that this node has a routing entry for the left base station and hence it sends a UNI_PASV_RESV message towards that base station. The flow of this unicast passive reservation message is shown by the yellow line in the figure. It also removes the address of the left base station from the NEIGHOURS object of the aggregate reservation message before forwarding it upstream. When the Cellular IP node 1 receives aggregate passive reservation message it repeats the above process viz. it sends a UNI_PASV_RESV message towards the third base station. After this, it removes the address of the 


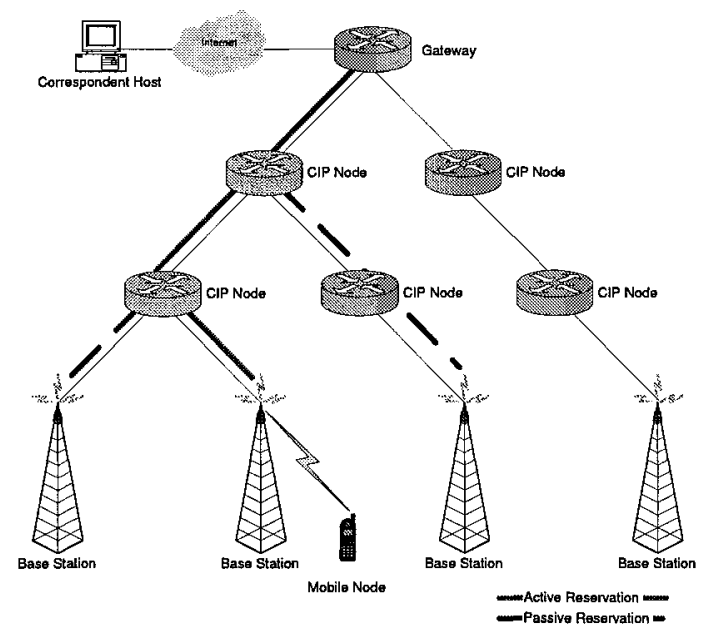

Figure 5. Reseravtion style in the Cellular IP Access Network.

third base station from the NEIGHOURS object which then becomes empty henceforth aggregate passive reservation message is no longer forwarded upstream.

Here the role of the base station specific routing should be clearly understood as without it routing the passive reservation messages to the base stations using conventional Cellular IP routing is not possible.

- The CIP node which needs to forward a UNI_PASV_RESV message on some interface makes passive reservation towards that interface. The base should make reservation on the wireless interface. The interface number is available to the RSVP daemon through RSRR from the CIP routing table which has entries for the required base stations.

In Fig. 6, CIP node 3 makes passive reservation (shown by the yellow line) towards the interface along which packet towards the first base station is sent. CIP node 1 and 4 makes passive reservation (shown by the blue line) towards the interface along which packets towards the third base station is sent.

- The RSVP daemon in the CIP nodes creates PATH and RESV states upon receiving the unicast passive reservation message. Note that the unicast passive reservation message carries all the necessary RSVP objects for establishing the PATH and RESV states. For PATH state the PHOP object always contains the address of the domain gateway router.

- The base stations on receiving the unicast passive reservation message reserves resources on their wireless interface. 


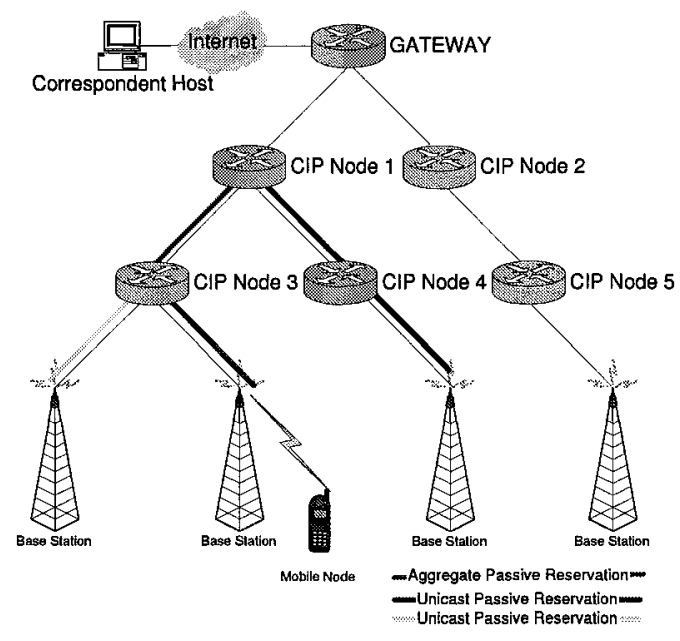

Figure 6. Initiating Passive Reservation from Base Station.

Mobile Node performs handoff. When the mobile node performs handoff it sends a semi-soft packet towards the gateway which configures routing cache with entries towards the new base station. As these entries are made in the routing cache:

- The routing daemon informs the RSVP daemon about the new entries through a RCN (Route Change Notification) using the RSRR interface. item On receiving the RCN message RSVP daemon activates the passive reservation meant for the mobile host.

- Because the cross over node does not forward the semi-soft packet further upstream only passive reservation up to the cross over node is activated.

On receiving a semi-soft handoff packet the base station RSVP daemon initiates passive reservation as described earlier. When CIP nodes receive the unicast passive reservation message for passive reservation along a link for the mobile node and they already have active reservation along that link for the mobile node then the RSVP daemon on these nodes simply convert the active reservation to passive reservation otherwise if no active reservations exist then new passive reservations are created. Fig. 7. illustrates changed reservation styles after handoff is performed by the mobile.

Mobile Node is Sender. When mobile node is the sender everything is same as explained above except that PHOP object content changes.

More specifically, when a mobile node is a sender it sends PATH message upstream. The PHOP object in these messages contains the address of the 


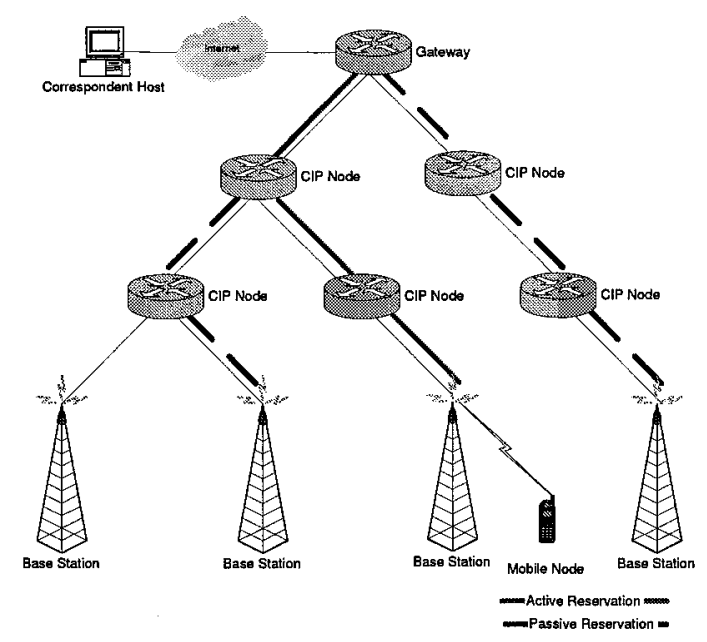

Figure 7. Changed reseravtion style in the Cellular IP Access Network after the Mobile Node handoffs.

mobile node. When a RESV message needs to be sent to the mobile it looks into the PHOP object in the PATH state for next hop address and gets the link (the link would be the one on which the packets destined for the mobile are forwarded) on which to be forwarded from the Cellular IP routing table through RSRR interface.

When the mobile node needs to send a semi-soft packet during handoff it actually sends a PATH and AGGR_PASV_RESV message upstream. Note the aggregate passive reservation. This is done so as to prevent signaling overheads in the access network. The base station thus initiates passive reservation

\section{Case II. Mobile Node is in Idle State.}

Mobile Node is Receiver. When mobile is in idle state any data meant for the mobile in Cellular IP access network forms an implicit paging packet i.e the mobile is delivered the packet using entries in paging and routing caches or using broadcast if entries does not exist. Their does not exist any explicit paging message to the mobile to wake it up from idle state.

When the gateway receives any PATH message for the mobile and the mobile node is determined to be in idle state then the gateway buffers the PATH message till the mobile node is paged to establish up-to-date routing entries in the routing caches. This prevents unnecessary broadcast of the PATH message in the network. 
Mobile Node is Sender. In this case since the mobile is itself doing a transmission (sending a PATH message) it changes from idle to active state.

Integrated Cellular IP and RSVP signaling in the access network. The mobile node needs to send CIP route refresh messages to keep up-to-date location information. Also, to prevent RSVP state established in the nodes along the path to the receiver from timing out mobile node also needs to refresh them periodically in case it is the sender. To prevent unnecessary signaling the RSVP refresh messages also refresh CIP routing and paging caches in the nodes and thus when RSVP refresh messages have been sent explicit CIP refresh messages need not be sent.

Proxy RSVP Signalling. There is a need to refresh passive reservations also otherwise they may timeout due to lack of refreshes. To refresh passive reservation we propose proxy RSVP signaling by the base station (with which the mobile is currently associated) on behalf of the mobile node. This base station sends proxy refresh messages to the base stations in the neighboring cell periodically. The time period is determined taking into account that this signaling does not generate too much control traffic and does not consume too much network bandwidth.

\section{Conclusion and Future Work}

Our proposal provides a signaling mechanism to reserve resources in the Cellular IP access network to provide fine grained QoS to service quality sensitive applications. This solution is fully distributed. The load is shared by all the Cellular IP nodes and the base stations rather than everything is done by the domain gateway router which increases the load on the gateway and introduces a single point of failure for resource reservation. Also, the use of proxy RSVP signaling by the base station on behalf of the mobile node to maintain passive reservation conserves battery power of the mobile node as well the precious wireless bandwidth.

Currently we are implementing the proposed scheme in the Network Simulator [11] for performance evaluation and investigating the use of mobility profile of the mobile node or statistical information about its mobility to decide about passive reservation to be done. This would allow passive reservation to be done towards fewer neighboring base stations rather than all.

\section{Acknowledgments}

The work presented in this paper was partially funded by the Centre for Information and Systems of the University of Coimbra and Portuguese Foundation for Science and Technology. 


\section{References}

[1] "Cellular IP" J. Gomez, A. T. Cambell, J. Gomez, Z.Turanyi, A. Valko, C-Y Wan, Work in Progress, <draft-valko-cellularip-01.txt>

[2] "Cellular IPv6", Shelby Z., Gatzounas D., Campbell A. \& Wan C-Y", IETF Internet Draft, draft-shelby-cellularipv6-01, July 2001.

[3] "Integrated Services in the Internet Architecture: an Overview," R. Braden, D. Clark, and S. Shenker: IETF RFC 1663, June 1994.

[4] "Resource ReServation Protocol (RSVP) Version 1 Functional Specifi cation," R. Braden, L.Zhang et al. IETF RFC 2205, September 1997.

[5] "MRSVP: A Reservation Protocol for an Integrated Services Packet Network with Mobile Hosts", A. K. Talukdar, B. R. Badrinath, and A. Acharya Tech. Rep Des-tr-337, Department of Computer Science, Rutgers University, U.S.A., 1997

[6] "Quality of Service Architecture for Wireless Networks: IntServ and DiffServ Models" I. Mahadevan and K. Sivalingam, I-SPAN, 1999.

[7] "An Experimental Architecture for providing QoS guarantees in Mobile Networks using RSVP", I. Mahadevan and K. Sivalingam, IEEE PIMRC, Boston, September 1998.

[8] "RSVP Mobility Support: A Signaling Protocol for Integrated Services Internet with Mobile Hosts", W.T. Chen et al, INFOCOM 2000.

[9] "Quality of Service guarantees in mobile computing", S. Singh, Computer Communications, vol. 19, pp. 359-371, Apr. 1996.

[10] "RSRR: A Routing Interface for RSVP", D. Zappala and J. Kann, Internet draft, June 1998.

[11] Network Simulator "http://www.isi.edu/nsnam/ns". 